\title{
A Bayesian framework to objectively combine metrics when developing stressor specific multimetric indicator
}

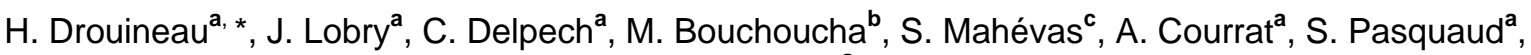 \\ M. Lepage ${ }^{a}$
}

\author{
${ }^{a}$ Cemagref - UR EPBX - EPBX, 50 av. de Verdun, Gazinet, 33612 CESTAS Cedex, France \\ b Ifremer - Laboratoire Environnement-Ressources, ZP de Bregaillon, BP n 330 - 83507 La Seyne sur Mer, \\ France \\ c Ifremer - Département EMH, rue de l'Ile d'Yeu, 44311 Nantes Cedex 03, France
}

\author{
*: Corresponding author : tel: +33 (0)5 57892708 ; fax: +33 (05)5 57890801 ;
}

email address : hilaire.drouineau@cemagref.fr

\begin{abstract}
:
In the context of the European Water Framework Directive (WFD), monitoring programs and related indicators have been developed to assess anthropogenic impacts on various components of aquatic ecosystems. While great precautions are usually taken when selecting and calculating relevant core metrics, little attention is generally paid to the generation of the multimetric indicator, i.e. the combination of the different core metrics. Indeed, most multimetric indicators are generated by simply averaging or summing metrics, without taking into account their sensitivity and their variability. Moreover, few indicators provide a rigorous estimate of the uncertainty of the assessments, while this estimation is essential for managers. In this context, we developed a Bayesian framework to build multimetric indicators aiming at improving those two weaknesses. This framework is based on two phases. First, pressure-impact statistical models are developed to quantify the impact of pressure on various fish metrics. Then the Bayesian theorem is applied to estimate probabilities of being at a certain anthropogenic pressure level from fish observation and pressure-impact models outputs. The Bayesian theorem allows to combine objectively the different core metrics, taking into account their sensitivity and their variability, and to provide rigorous uncertainty quantification, which is especially valuable in the WFD context.
\end{abstract}

The method is applied as illustrative example on transitional French water bodies to demonstrate its relevance, especially in the Water Framework Directive context though the method is generic enough to be applied in various contexts.

Keywords : Multimetric fish-based indicator; Bayesian method; Pressure-impact models; Water Framework Directive; Anthropogenic pressure; Monitoring program; Transitional waters 


\section{Introduction}

Multimetric fish-based indicators are often used to assess the ecological quality of aquatic ecosystems (Hughes and Oberdorff, 1999). Ideally, they are based on a set of non-redundant core metrics measured on fish assemblages. The main interest of such a tool is to provide an assessment that integrates several aspects of the fish assemblages through the different core metrics (Karr and Chu, 1999). Moreover, multimetric indicators are often considered as more sensitive and robust indicators of ecological quality than any of the individual metrics selected for their construction (Deegan et al., 1997; Hughes et al., 1998; Karr and Chu, 1999). A specific class of multimetrics indicator, called stressor-specific multimetrics indicator by Hering et al. (2006), is designed to detect the impact of a specific stressor on the ecosystem.

This approach has been widely used in the context of the Water Framework Directive (European Water Framework Directive 2000/60/EC; WFD) to detect the impact of anthropogenic pressures on riverine and estuarine fish assemblages (Borja et al., 2004; Breine et al., in Press; Breine et al., 2007; Coates et al., 2007; Delpech et al., 2010; Franco et al., 2009; Martinho et al., 2008; Pont et al., 2009; Pont et al., 2006; Uriarte and Borja, 2009).

Hering et al. (2006) presented a "cook-book" to develop multimetric indicators that distinguishes 6 main steps: (i) selection of the form of the multimetric indicator (general or stressor specific), (ii) metric selection, (iii) metric calculation, (iv) multimetric indicator generation by combining the different metrics, (v) setting class boundaries and (vi) results interpretation. One of the main difficulties rests in step (iv): the indicator should objectively combine the different metrics and, in the case of a stressor specific indicator, it should take into account the sensitivity of the metric to the stressor. Additionally, a measure of uncertainty should be calculated, since bioassessements have little value without any uncertainty measures (Kurtz et al., 2001; Clarke and Hering, 2006; Clarke et al., 2006; Carstensen, 2007; Beliaeff and Pelletier, 2011). Uncertainty measure is explicitly required by the WFD (European Communities, 2004). More specifically, it is of particular importance to be able to quantify the probability to don't be at least in "good" status class or not since the directive aims at achieving "good" water status for all water bodies.

Several approaches have been proposed to combine the core metrics. Delpech et al. (2010), for example, scored each metric independently; the final score being the average of the individual scores. Breine et al. (2007) proposed a statistical method to minimise type I and type II classification error. Pont et al. (2006) used reference sites to build a theoretical distribution of metrics in a reference state and developed a probabilistic framework to combine the metrics measured on other sites by calculating probabilities to belong to the reference distribution.

The aim of this paper is to propose a new approach that provides an original Bayesian framework to combine objectively the different metrics. The approach is based on two phases, which corresponds respectively to steps (ii) to (iii) and to step (iv) in Hering et al. (2006). In the first phase, pressure-impact models similar to those developed by Courrat et al. (2009) and Delpech et al. (2010) are developed. They enable objective selection and calculation of core metrics (steps (ii) and (iii) in Hering et al. (2006)). Then in the second phase, outputs of the models are used to compute probabilities of the ecosystem experiencing particular anthropogenic pressures levels by applying the Bayesian framework (step (iv) in Hering et al. (2006)). In this phase, the method used to combine metrics takes into account their sensitivity to the stressor and their variability. By providing a measure of uncertainty as required in the WFD context (European Communities, 2004), this method represents a great improvement with respect to existing methods, and is a great advance regarding the fourth step described 
by Hering et al. (2006) for the construction of multimetric indicators. As an illustrative example, the method is applied to French Mediterranean lagoons, classified as transitional waters for the WFD.

\section{Material and methods}

\subsection{Methods: description of the original approach}

Hering et al. (2006) cook book describes six main steps when developing a multimetric indicator. The method presented in this paper is partially based on Courrat et al. (2009) and Delpech et al. (2009), consequently, only the original part of the method, aiming at improving steps (ii) to (iv) from Hering et al. (2006), will be presented here.

2.1.1. First phase: selection of candidate metrics using pressure-impact models (step (ii) to (iii) in Herring et al. (2006))

The idea when building a multimetric stressor-specific indicator is to select metrics among a list of candidate metrics and to combine them in an indicator to detect a gradient of stressor (Hering et al., 2006). To build the indicator, a dataset with various candidate metrics (for example fish densities, number of species...) in columns measured on several fishing operations (in rows) is generally available. A measure or a proxy of the stressor is also required to check the correlations between the indicator and the stressor (Hering et al., 2006).

Pressure-impact approach (Courrat et al., 2009; Delpech et al., 2010) consists in developing pressure-impact statistical models by fitting generalized linear models (GLMs) that describe the impact of the stressor on the different candidate metrics, taking into account other covariates such as the variability due to the sampling procedure or to environmental characteristics. This approach is relevant to select candidate metrics since only metrics that are significantly impacted by the stressor are selected. The GLMs can be written on the matrix form:

(1) $g^{(j)}\left(E\left(M^{(j)}\right)\right)=\alpha^{(j)} \cdot X+\beta^{(j)} \cdot \operatorname{Pr}$

with $g^{(j)}\left(E\left(M^{(j)}\right)\right)$ the link transformed (function $g^{(j)}$ ) expected value of the $j$-th metric, $\alpha^{(j)}$ the regression parameters for covariables, $X$ the model matrix for the covariables, $\beta^{(j)}$ the regression parameter for the stressor and $\operatorname{Pr}$ the vector of stressor values.

Candidate metrics for which $\beta^{(j)}$ are significantly different from zero, i.e. significantly impacted by the stressor, are potentially relevant metrics to include in the indicator.

2.1.2. Second phase: computing probabilities of pressure levels given observed metrics (step (iv) in Herring et al. (2006))

The aim of a stressor specific multimetric fish-based indicator is to evaluate the level of a specific stressor by observing metrics describing the fish assemblage given particular stressor-metric relationships (Hering et al., 2006). To fulfil this objective, the present method uses the pressure-impact statistical models described above incorporating their results in a Bayesian framework. Applying the Bayes theorem makes possible to calculate the probability that a waterbody is in an ecological quality class given the fish data. GLMs likelihood functions 
are used to convert fish observations into probability densities; so that metrics can be combined on a common scale. Probability densities account for both the uncertainty of the model (through variance functions of the GLMs) and the sensitivity of the metric to the stressor (through the values of regression parameters).

The probability that the pressure level $\operatorname{Pr}$ is comprised within a given range $\left[p_{1}, p_{2}\right]$ given $I$ new fishing operations (denoted $1, \ldots, i, \ldots, I)$, which corresponds to an a posteriori probability in a Bayesian framework, is:

(2) $p\left(p_{1} \leq \operatorname{Pr}<p_{2} \mid M^{(1)}=\left\{m_{1}^{(1)}, \ldots, m_{n}^{(1)}\right\}, \ldots, M^{(J)}=\left\{m_{1}^{(J)}, \ldots, m_{n}^{(J)}\right\}\right)$

with $m_{i}^{(j)}$ observed values of the metric $j$ (from 1 to $J$ ) during fishing operation $i$ (from 1 to $n$ ).

The probability is equal to (cf. Annex):

(3)

$$
p\left(p_{1} \leq \operatorname{Pr}<p_{2} \mid M=m\right)=\frac{\iint_{\beta} \int_{p_{1}}^{p_{2}}\left[\prod_{i} \prod_{j} f_{M}(j)_{\mid P r}\left(p, m_{i}^{(j)}\right) \cdot f_{P r}(p) \cdot f_{A, B}(\alpha, \beta)\right] d p \cdot d \alpha \cdot d \beta}{\iint_{\alpha} \int_{p_{\min }}^{p_{\max }}\left[\prod_{i} \prod_{j} f_{M^{(j)} \mid \operatorname{Pr}}\left(p, m_{i}^{(j)}\right) \cdot f_{P r}(p) \cdot f_{A, B}(\alpha, \beta)\right] d p \cdot d \alpha \cdot d \beta}
$$

with $\left[p_{\min }, p_{\max }\right]$ the domain of definition of the stressor. $f_{M}^{(j)_{\mid P r, A, B}}\left(p, m_{i}^{(j)}, \alpha, \beta\right)$ is the density of probability of an observation given the matrix model and the regression parameters. It corresponds to the likelihood of an observation in the GLMs, so that it can be directly calculated as an output of the pressure-impact models (McCullagh and Nelder, 1989). $f_{A, B}(\alpha, \beta)$ corresponds to a prior on the distribution of the regression parameters. Regression parameters' estimates follow a multinomial distribution when fitting a model by maximum likelihood (such as GLMs), consequently a multinormal (or multistudent for small datasets) prior using GLMs estimates and corresponding variance-covariance matrix $\Sigma$ can be used: $\{A, B\} \sim N(\{\hat{\alpha}, \hat{\beta}\}, \Sigma) . f_{P r}(p)$ is the equivalent of a prior in a Bayesian framework and enables to include expert knowledge.

In the absence of precise knowledge, uninformative uniform prior may be used and if standard error of regression parameters estimates are small compared to the GLMs dispersion parameters, equation 3 can be approximated by:

(4) $p\left(p_{1} \leq \operatorname{Pr}<p_{2} \mid M=m\right)=\frac{\int_{p_{1}}^{p_{2}}\left[\prod_{i} \prod_{j} f_{M}(j)_{\mid P r}\left(p, m_{i}^{(j)}\right) \cdot f_{P r}(p)\right] d p}{\int_{p_{\min }}^{p_{\max }}\left[\prod_{i} \prod_{j} f_{M}(j)_{\mid P r}\left(p, m_{i}^{(j)}\right) \cdot f_{P r}(p)\right] d p}$

However any expert knowledge or meta-analysis results can be used to build more informative priors on the stressor, and priors on the regression parameters can be easily incorporated if estimation error are significant compared to observation errors. 
By using this formula, the probability of being in any range of stressor can be easily calculated.

\subsection{Illustrative example}

In this illustrative example, the method is applied to generate a multimetric fish-based indicator for detecting anthropogenic pressure in lagoons.

\subsubsection{Fish data and anthropogenic pressure index}

Fourteen lagoons along the French Mediterranean coast are considered in this study (Table 1, Fig. 1). Lagoons were described by two physical factors (Table 1) that have proved to have a decisive effect on fish assemblages in lagoons (Perez-Ruzafa et al. 2007):

Surf: total surface area $\left(\mathrm{km}^{2}\right)$

Sect: channels cross-sectional area 1, 2, 3... $\left(\mathrm{m}^{2}\right)$

A dataset with 348 fishing operations (a fishing operation corresponds to a fyke-net fishing during 24 hours) carried out in the context of the Water Framework Directive was available. Fishing operations were conducted in spring and autumn using fyke nets in shallow water (between $0.5 \mathrm{~m}$ and $2.5 \mathrm{~m}$ ).

Each captured fish was identified at the species level, and then assigned to different functional guilds according to Delpech et al. (2010).

An anthropogenic pressure index was estimated using an approach similar to Courrat et al. (2009) and Delpech et al. (2010). A principal component analysis (PCA) was carried out on mean concentrations of four heavy metals $(\mathrm{Cd}, \mathrm{Zn}, \mathrm{Cu}, \mathrm{Pb})$ and one organic contaminant (S16HAPs) provided by the RINBIO biointegrator network set up by IFREMER (Andral et al., 2004) in twelve of the lagoons (data were not available for Biguglia and Grand Bagnas - Fig. 1). All contaminants showed a strong correlation with the first axis of the PCA which represents $55 \%$ of the total variance. We thus used lagoons coordinates on this axis as a measure of contamination (Fig. 2). As suggested in Courrat et al. (2009) and Delpech et al. (2010) for French estuarine areas, we considered contamination as a proxy of global anthropogenic pressure in each lagoon.

The RINBIO network also provides quality thresholds for each contaminant. Therefore it was possible to determine the thresholds of five quality classes on our anthropogenic pressure index by projecting the contaminant thresholds on the PCA factorial map (Fig. 2, Table 2). Considering this method, the twelve lagoons were in the two best quality classes.

\subsubsection{Candidate metrics and pressure-impact models}

Several metrics were calculated for each fishing operation (Table 3): total abundance, total species richness, total number of fishes per guild, number of distinct species per guild. Total number of fishes were log-transformed $(\log (x+1))$ to limit the influence of outliers (fishing events with high catches). Metrics were computed at the fishing operation level rather than at a larger scale in order to take into account the metric variability due to sampling protocol (Courrat et al., 2009; Delpech et al., 2010). 
GLMs were built for each metric (Table 3). A stepwise backward procedure was used to select the most relevant and parsimonious models based on the corrected Akaike Information Criterion (AICC), which is for small datasets (Burnham and Anderson, 2002). Such pressureimpact models were fitted on data from the twelve lagoons monitored by the RINBIO network.

\subsubsection{Multimetric indicator generation and probabilities computation}

Graphical analysis of the residuals was carried out to check that GLMs assumptions were respected. In that case, standardised residuals of deviance were normally distributed (McCullagh and Nelder, 1989) so Pearson's correlation coefficients are appropriate to analyse residuals independence between core metrics.

After checking metric correlations, all 348 fishing operations from all the 14 lagoons (including lagoons in which pressure index was not available) were used to compute the probability that each lagoon belong to each stressor quality class using equation 4.

Results obtained with the above described dataset are presented here only as an illustrative example of possible outputs of the method given a plausible use in a WFD context.

\section{Results}

\subsection{Selection of core metrics}

In the illustrative example presented here, four metrics responded as expected to an increase of the anthropogenic pressure index (Table 4). Values of estimated regression parameters for the pressure index were low (Table 4 ) but significantly different from 0 . Both abundance metrics (TD and $D M)$ and species richness metrics (SR and NM) were significantly "impacted" by the pressure index. Moreover, both pressure-impact models at the functional group level $(D M$ and $N M)$ and at the fish assemblage levels (TD and $S R$ ) were significant.

Graphical analysis of the residuals confirmed that GLMs assumptions were respected (consistence with the assumed distribution, with the assumed mean-variance relationship and independence). Correlation analysis tests theoretically rejected the independence assumptions. However, Pearson's correlation coefficients for the three metrics $T D, D M$ and $S R$ were very low (inferior to 0.24 ) and independence can be reasonably assumed.

Since standard-errors were small compared to observation errors and no expert knowledge was available, equation 4 rather than equation 3 was used to compute probabilities of being in a given pressure range.

\subsection{Ecological quality assessment}

For each of the 14 surveyed lagoons, the posterior probability to be in each of the five quality classes given the observed fish metrics were computed using equation 4 (Fig. 3). Moreover, the fish indicator was very discriminant: for all lagoons, the most probable ecological quality class was superior $>60 \%$, except for Berre where we could not distinguish between good and very good classes.

Results were also analysed metric by metric (Fig. 4). For example, observed $T D$ was relatively low in Or but high in Vaccarès compared to what was expected given the anthropogenic 
pressure index. Results at the core metrics level also illustrate that diagnostics based on a single metric are much more variable than results of the multimetric indicator; some individual metrics indicating medium or poor quality in some lagoons (for example, $D M$ and $R T$ in Biguglia).

\section{Discussion}

\subsection{Comments related specifically to the illustrative example}

The ecological quality of 14 French Mediterranean lagoons was assessed as an illustrative example, and further analysis should be carried out to improve the current application before using this multimetric fish indicator for management. More specifically, the present pressure index should be improved. Indeed, there was no available measure for oligohaline lagoons, and values for other lagoons were not really discriminant: they were all in the two best quality classes according to the pressure index leading to a dataset without much contrast. Moreover, available data were still limited (though they will increase with the implementation of WFD routine surveys) so it was necessary to use the same fishing observations for both GLMs construction and probabilities calculations (except for Biguglia and Grand Bagnas) whereas theoretically, the dataset used to build pressure-impact models should be distinct from the dataset used in the Bayesian calculation. Consequently, this paper will focus on the method used to build the indicator rather than on the illustrative example. So, specific results on lagoons or classification will not be discussed. However, with few modifications, the method will be relevant to assess the ecological status of those water bodies.

\subsection{Methodological aspects}

The approach proposed by Delpech et al. (2010) based on pressure-impact statistical models is especially relevant to describe the impact of a specific stressor such as anthropogenic pressure, on fish assemblages. However, the method to generate the multimetric indicator by combining the core metrics was rather subjective and did not provide any uncertainty quantification (Brind'Amour and Lobry, 2009). The aim of this paper was to propose a method to construct multimetric indicators that provide an objective scheme for combining metrics based on a Bayesian framework. The first phase is to develop pressure-impact GLMs (Courrat et al., 2009; Delpech et al., 2010) or any other models (general additive models, mixed models...) that provides a likelihood measure. GLMs outputs are then used to calculate probabilities that a water body belongs to an ecological quality class from fish observations. The present method presents some similarities with Bayesian discriminant analysis (Geisser, 1964; Keehn, 1965) since both methods compute probabilities of being in one pre-defined. However, our approach relies on the construction of pressure-impact models in a first phase, that allows a great variability on the form of the distribution of the metric given the pressure and the regression model, and to easily account for environmental covariates. Moreover, this first phase provides valuable information at the metric level that can be analysed in the light of ecological concepts (e.g. (Nicolas et al., 2010a; Nicolas et al., 2010b) for examples with fish data on estuaries), rather than building discriminant functions that are only based on a statistical criterion and are often less flexible.

One of the main advantages of the method is that it combines core metrics taking into account both (i) the sensitivity of the metric to the stressor through the regression parameter in the pressure-impact statistical model and (ii) the uncertainty of the statistical model through the variance function of the GLMs included in the likelihood computation (equation 4). Furthermore, while Delpech et al. (2010) couldn't include any metric based on species richness in their final multimetric indicator because they were not powerful discriminant 
metrics, the present method can include them and incorporate their weak, though perhaps important, discriminant ability in the collective. The method also provides a measure of uncertainty by computing posterior probabilities that the water body is in each pre-defined quality class (Fig. 3). This uncertainty estimation is especially important for managers and is required by the WFD (European Communities, 2004). More specifically, the fact that it is possible to estimate the for a water body to be "good or better" ecological status or not is especially important given the importance of this threshold in the directive. This particular aspect is probably the greatest advantage of this method.

Moreover, in this approach, the problem is considered in a different way to what is usually done. Generally, pressure indices are used to predict expected fish assemblages metrics values at various pressure levels in order to estimate metric thresholds (Courrat et al., 2009; Delpech et al., 2010). In the present approach, the method is similar in a first phase (development of pressure-impact statistical models), but then the problem is reversed by trying to predict a level of pressure from fish metrics so that it is possible to define a priori pressure thresholds. This approach seems much more relevant in an operational monitoring context when stakeholders have to take appropriate management decisions based upon the latest observations of ecological state.

As mentioned by Hughes et al. (1998) and Karr \& Chu (1999), multimetric indices are more robust than single metrics because the metrics combination generally makes the indicators less variable than each core metric considered individually. This is illustrated by the higher variability of the results when providing a diagnostic for each metric (Fig. 4) compared to the results when applying the global multimetric indicator (Fig. 3).

An other important aspect to consider when developing an indicator is the consequences of misclassification. For example, in the illustrative example, three lagoons were misclassified by the fish-based indicator with respect to the pressure index. Three main reasons may explain this result: (i) the natural variability of biological measures (however the sampling procedure is designed to minimize this aspect), (ii) pressure index was not perfectly relevant since it is only a proxy of global anthropogenic pressures or (iii) lagoons are more or less resilient with respect to anthropogenic pressure. The two last points are especially interesting. The fish indicator for Or Lagoon is pessimistic compared to the pressure index because TD is low compared to the pressure-impact model prediction, i.e. it seems that Or is especially sensitive to an increase of pressure or that pressure index was underestimated. On the other hand, the fish indicator provides a more pessimistic diagnostic for Vaccarès than the pressure index because $T D$ is high compared to what is predicted by the pressure-impact model, possibly because Vaccarès is less sensitive or because the pressure index was overestimated for this lagoon. Consequently, the indicator tends to penalize lagoons which are more sensitive to pressure and/or to consistently deal with pressure index misspecification, which is consistent with the precautionary approach.

Another advantage of the Bayesian approach relies on the possibility to include prior knowledge in the indicator. Expert knowledge or results of independent analysis can be used to build informative prior. That knowledge is difficult to incorporate in traditional methods. Yet, it is interesting to include it by building a priori pressure density distribution which would influence the results (equation 3 ).

Moreover, in the absence of previous expert knowledge and when standard-errors of the GLMs are small compared to dispersion as it is in the illustrative example, equation 4 can be used rather than equation 3 . Since equation 4 consists in a single integral, it is possible to compute the probabilities using usual numerical integration algorithm. However, in most situations, this solution will not be possible, and Bayesian inference algorithm, such as Gibbs 
Samplings will be appropriate. In that case, the use of traditional Bayesian diagnostic tools will of course be necessary to check relevance of the results, and more specifically the convergence of the algorithm.

Finally, the present method is completely generic and can be implemented in many situations, as long as it is possible to build pressure-impact statistical models (enough data and, sufficient knowledge on determinant abiotic factors). More specifically, any kind of metrics can be included in the indicator: density, numbers, proportion (GLM with binomial family) or other metrics as soon as likelihood of new observations given a pressure can be calculated (the principle limitation will generally concern the number of available data to fit pressure impactmodels). Consequently, it may be applied for many other stressor-specific indicators, especially indicators developed in the WFD context. However, a difference with traditional indicators is that generally, a set of thresholds are defined for each metric in order to get a score by metric which are then aggregated to get the assessment, whereas in our approach, only one set of threshold is required and is directly defined on the pressure index. Consequently, ecological quality ratio (EQR, the WFD defined EQR as a ratio between the actual level of an indicator and the reference level of the indicator) does not have exactly the same meaning as in other indicators. However, this approach is more consistent with the initial objective of a multimetric indicator, i.e. assessing levels of pressure by analysing metrics, and more consistent with the approach recommended in the annexes III and IV of the WFD guidance document (European Communities, 2009).

\section{Acknowledgements}

We want to acknowledge Dr Patrick Lambert and Dr Daniel Duplisea for fruitful discussions on the method. We also would like to thank the Rhone-Mediterranean and Corsica Water Agency for their collaboration and their support in the data collection. This work is part of the WISER European project (Water bodies in Europe: Integrative Systems to assess Ecological status and Recovery) funded by the European Union under the 7th Framework Programme, Theme 6 (Environment including Climate Change) (contract No. 226273), http://www.wiser.eu.

\section{References}

Andral, B., Stanisiere, J.Y., Sauzade, D., Damier, E., Thebault, H., Galgani, F., Boissery, P., 2004. Monitoring chemical contamination levels in the Mediterranean based on the use of mussel caging. Mar. Pollut. Bull. 49: 704-712.

Beliaeff, B., Pelletier, D., 2011. A general framework for indicator design and use with application to the assessment of coastal water quality and marine protected area management. Ocean \& Coastal Management 54: 84-92.

Borja, Á., Franco, J., Valencia, V., Bald, J., Muxika, I., Belzunce, M.J., Solaun, O., 2004. Implementation of the European water framework directive from the Basque country (northern Spain): a methodological approach. Mar. Pollut. Bull. 48: 209-218.

Breine, J., Quataert, P., Stevens, M., Ollevier, F., Volckaert, F., Van den Bergh, E., Maes, J., in Press. A zone-specific fish-based biotic index as a management tool for the Zeeschelde estuary (Belgium). Mar. Pollut. Bull. 
Breine, J.J., Maes, J., Quataert, P., Van den Bergh, E., Simoens, I., Van Thuyne, G., Belpaire, C., 2007. A fish-based assessment tool for the ecological quality of the brackish Schelde estuary in Flanders (Belgium). Hydrobiologia. 575: 141.

Brind'Amour, A., Lobry, J., 2009. Assessment of the ecological status of coastal areas and estuaries in France, using multiple fish-based indicators: a comparative analysis on the Vilaine estuary. Aquat. Living Resour. 22: 559-572.

Burnham, K.P., Anderson, D.R., 2002. Model Selection and Multimodel Inference: A Practical Information Theoretic Approach. Springer, New York.

Carstensen, J., 2007. Statistical principles for ecological status classification of Water Framework Directive monitoring data. Mar. Pollut. Bull. 55: 3-15.

Clarke, R., Davy-Bowker, J., Sandin, L., Friberg, N., Johnson, R., Bis, B. 2006. Estimates and comparisons of the effects of sampling variation using 'national' macroinvertebrate sampling protocols on the precision of metrics used to assess ecological status. Hydrobiologia 566: 477-503.

Clarke, R., Hering, D. 2006. Errors and uncertainty in bioassessment methods - Major results and conclusions from the STAR project and their application using STARBUGS. Hydrobiologia 566: 433-439.

Coates, S., Waugh, A., Anwar, A., Robson, M., 2007. Efficacy of a multi-metric fish index as an analysis tool for the transitional fish component of the Water Framework Directive. Mar. Pollut. Bull. 55: 225-240.

Courrat, A., Lobry, J., Nicolas, D., Laffargue, P., Amara, R., Lepage, M., Girardin, M., Le Pape, O., 2009. Anthropogenic disturbance on nursery function of estuarine areas for marine species. Estuarine, Coastal and Shelf Science. 81: 179-190.

Deegan, L.A., Finn, J.T., Ayvazian, S.G., Ryder-Kieffer, C.A., Buonaccorsi, J., 1997. Development and validation of an estuarine biotic integrity index. Estuaries. 20: 601-617.

Delpech, C., Courrat, A., Pasquaud, S., Lobry, J., Le Pape, O., Nicolas, D., Boët, P., Girardin, M., Lepage, M., 2010. Development of a fish-based index to assess the ecological quality of transitional waters: The case of French estuaries. Mar. Pollut. Bull. 60: 908-918.

European Communities, 2004. Common Implementation Strategy for the Water Framework Directive (2000/60/EC). WFD CIS Guidance Document No7, European Communities, EC, Luxembourg, 153p.

European Communities, 2009. Common Implementation Strategy for the Water Framework Directive (2000/60/EC). WFD CIS Guidance Document No14, European Communities, EC, Luxembourg, 55p.

Franco, A., Torricelli, P., Franzoi, P., 2009. A habitat-specific fish-based approach to assess the ecological status of Mediterranean coastal lagoons. Mar. Pollut. Bull. 58: 1704-1717.

Geisser, S., 1964. Posterior odds for multivariate normal distributions. Journal of the Royal Society Series B: Methodological. 26: 69-76. 
Hering, D., Feld, C., Moog, O., Ofenböck, T., 2006. Cook book for the development of a Multimetric Index for biological condition of aquatic ecosystems: Experiences from the European AQEM and STAR projects and related initiatives. Hydrobiologia. 566: 311-324.

Hughes, R.M., Kaufmann, P.R., Herlihy, A.T., Kincaid, T.M., Reynolds, L., Larsen, D.P., 1998. A process for developing and evaluating indices of fish assemblage integrity. Canadian Journal of Fisheries and Aquatic Sciences. 55: 1618-1631.

Hughes, R.M., Oberdorff, T., 1999.Applications of IBI concepts and metrics to waters outside the United States, in: Simon, T.P. (Ed.), Assessing the Sustainability and Biological Integrity of Water Resource Quality Using Fish Communities, CRC Press, Boca Raton, Florida, pp. 7996.

Karr, J.R., Chu, E.W., 1999. Restoring life in running waters: better biological monitoring. Island Press, Washington DC.

Keehn, D.G., 1965. A note on learning for Gaussian properties. IEEE Trans. on Information Theory. 11: 126-132.

Kurtz, J.C., Jackson, L.E., Fisher, W.S., 2001. "Strategies for evaluating indicators based on guidelines from the Environmental Protection Agency's Office of Research and Development". Ecol. Ind. 1: 49-60.

Martinho, F., Viegas, I., Dolbeth, M., Leitão, R., Cabral, H., Pardal, M., 2008. Assessing estuarine environmental quality using fish-based indices: Performance evaluation under climatic instability. Mar. Pollut. Bull. 56: 1834-1843.

McCullagh, P., Nelder, J.A., 1989. Generalized Linear Models. Chapman \& Hall, London.

Nicolas, D., Lobry, J., Le Pape, O., Boët, P., 2010a. Functional diversity in European estuaries: Relating the composition of fish assemblages to the abiotic environment. Estuarine, Coastal and Shelf Science. 88: 329-338.

Nicolas, D., Lobry, J., Lepage, M., Sautour, B., Le Pape, O., Cabral, H., Uriarte, A., Boët, P., 2010b. Fish under influence: A macroecological analysis of relations between fish species richness and environmental gradients among European tidal estuaries. Estuarine, Coastal and Shelf Science. 86: 137-147.

Perez-Ruzafa A., Mompean M.C., Marcos C., 2007. Hydrographic, geomorphologic and fish assemblage relationships in coastal lagoons. Hydrobiologia 577:107-125

Pont, D., Hughes, R.M., Whittier, T.R., Schmutz, S., 2009. A Predictive Index of Biotic Integrity Model for Aquatic-Vertebrate Assemblages of Western US Streams. Transactions of the American Fisheries Society. 138: 292-305.

Pont, D., Hugueny, B., Beier, U., Goffaux, D., Melcher, A., Noble, R., Rogers, C., Roset, N., Schmutz, S., 2006. Assessing river biotic condition at a continental scale: a European approach using functional metrics and fish assemblages. Journal of Applied Ecology. 43: 7080.

Uriarte, A., Borja, A., 2009. Assessing fish quality status in transitional waters, within the European Water Framework Directive: Setting boundary classes and responding to anthropogenic pressures. Estuarine, Coastal and Shelf Science. 82: 214-224. 


\section{Annex: demonstration of the probability computation}

The objective of a stressor specific multimetric index is to detect the effect of the stressor by observing some metrics on the fish assemblage. Consequently, we are interesting in computing the probability that the pressure is included in a certain range given I new fishing operations (denoted $1, \ldots, i, \ldots, l)$ :

(A1) $p\left(p_{1} \leq \operatorname{Pr}<p_{2} \mid M^{(1)}=\left\{m_{1}^{(1)}, \ldots, m_{n}^{(1)}\right\}, \ldots, M^{(J)}=\left\{m_{1}^{(J)}, \ldots, m_{n}^{(J)}\right\}\right)$

with $m_{i}^{(j)}$ the value of metric $j$ measured in the $i$-th fishing operation, $n$ the number of fishing operation, $J$ the number of selected metrics.

We use the following notations

$M^{(j)}$ : random variable representing metric $j$ (from 1 to $\mathrm{J}$ )

$m_{i}^{(j)}$ : value metric $j$ in fishing operation $i$ (from 1 to $n$ )

Pr: random variable of the pressure index

$p_{1}$ and $p_{2}$ : thresholds of an ecological quality class corresponding to thresholds on the pressure index

$f_{X}$ : density function of random variable $X$

$A$ and $B$ random variables corresponding to the GLM regression parameters

By definition of a density function, we have

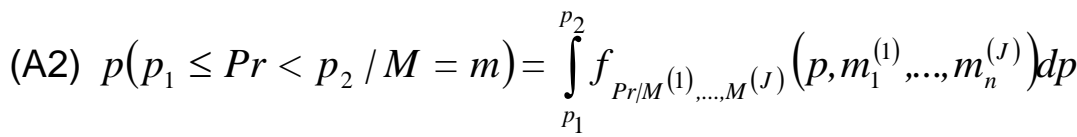

with $f_{\operatorname{Pr|M}(1), \ldots, M}(J)$ the density function of the variable $\operatorname{Pr} \mid M^{(1)}, \ldots, M^{(J)}$. Given that a conditional density is equal to the ratio of the joint density function over the marginal density.

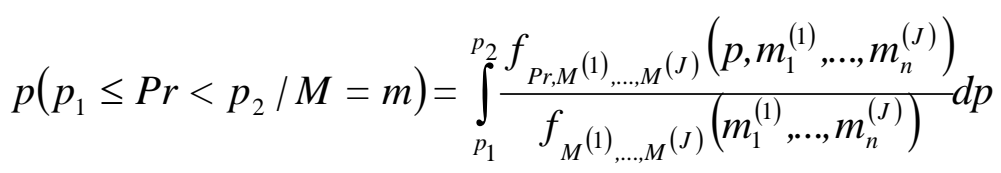

(A3)

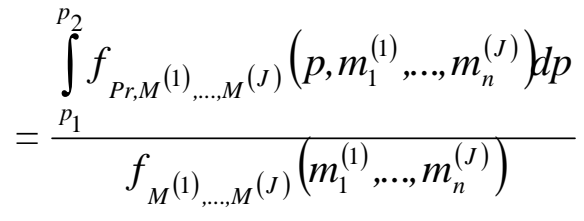


Because of the total conditional probability:

(A4) $p\left(p_{1} \leq \operatorname{Pr}<p_{2} \mid M=m\right)=\frac{\int_{p_{1}}^{p_{2}} f_{P r, M}{ }_{p_{2}, \ldots, M}^{(J)}\left(p, m_{1}^{(1)}, \ldots, m_{n}^{(J)}\right) d p}{\int_{p_{\min }}^{p_{\max }} f_{M^{(1)}, \ldots, M}(J){ }_{\mid P r}\left(p, m_{1}^{(1)}, \ldots, m_{n}^{(J)}\right) \cdot f_{P r}(p) d p}$

with $p_{\min }$ and $p_{\max }$, the minimum and maximum value of the pressure index.

Given that a conditional density is equal to the ratio of the joint density function over the marginal density.

(A5) $p\left(p_{1} \leq \operatorname{Pr}<p_{2} \mid M=m\right)=\frac{\int_{p_{1}}^{p_{2}} f_{M^{(1)}, \ldots, M}^{(J)_{\mid P r}}\left(p, m_{1}^{(1)}, \ldots, m_{n}^{(J)}\right) \cdot f_{P r}(p) d p}{\int_{p_{\min }}^{p_{\max }} f_{M^{(1)}, \ldots, M}(J)_{\mid P r}\left(p, m_{1}^{(1)}, \ldots, m_{n}^{(J)}\right) \cdot f_{P r}(p) d p}$

If the observations are independent:

(A6) $p\left(p_{1} \leq \operatorname{Pr}<p_{2} \mid M=m\right)=\frac{\int_{p_{1}}^{p_{2}}\left[\prod_{i}\left[\prod_{j} f_{M}(j)_{\mid P r}\left(p, m_{i}^{(j)}\right) \cdot f_{P r}(p)\right] d p\right.}{\int_{p_{\min }}^{p_{\max }}\left[\prod_{i} \prod_{j} f_{M}(j)_{\mid P r}\left(p, m_{i}^{(j)}\right) \cdot f_{P r}(p)\right] d p}$

GLMs provides a measure of $f_{M^{(j)} \mid P r, A, B}\left(p, m_{i}^{(j)}, \alpha, \beta\right)$, therefore we reformulate equation A6 as (A7)

$$
p\left(p_{1} \leq \operatorname{Pr}<p_{2} \mid M=m\right)=\frac{\iint_{\alpha} \int_{p_{1}}^{p_{2}}\left[\prod_{i} \prod_{j} f_{M}(j)_{\mid P r}\left(p, m_{i}^{(j)}\right) \cdot f_{P r}(p) \cdot f_{A, B}(\alpha, \beta)\right] d p \cdot d \alpha \cdot d \beta}{\iint_{\alpha} \int_{p_{\min }}^{p_{\max }}\left[\prod_{i} \prod_{j} f_{M^{(j)} \mid \operatorname{Pr}}\left(p, m_{i}^{(j)}\right) \cdot f_{P r}(p) \cdot f_{A, B}(\alpha, \beta)\right] d p \cdot d \alpha \cdot d \beta}
$$




\section{Tables}

Table 1. Main characteristics of the different lagoons, which are located along the French Mediterranean coast (Fig. 1) and number of fishing operations per season (a fishing operation corresponds to a fyke-net fishing during 24 hours).

Surf: lagoon surface $\left(\mathrm{km}^{2}\right)$. Sect: cross-sectional area of the inlets $\left(\mathrm{m}^{2}\right)$.

Grand Bagnas and Méjean does not have inlet directly connected to the sea

\begin{tabular}{lllll}
\hline & & & \multicolumn{2}{c}{ Number of fishing operations } \\
Lagoon & Surf & Sect & Spring & Summer \\
\hline Bages-Sigean & 38 & 600 & 12 & 15 \\
Berre & 133 & 517 & 15 & 15 \\
Biguglia & 14 & 3 & 8 & 8 \\
Diana & 5 & 61 & 8 & 10 \\
Grand Bagnas & 2 & 0 & 15 & 16 \\
La Palme & 5 & 25 & 16 & 16 \\
Méjean & 7 & 0 & 8 & 8 \\
Or & 33 & 15 & 7 & 8 \\
Palo & 1 & 10 & 8 & 8 \\
Prévost & 3 & 53 & 8 & 8 \\
Salses-Leucate & 54 & 367 & 23 & 29 \\
Thau & 69 & 237 & 30 & 15 \\
Urbino & 8 & 31 & 8 & 12 \\
Vaccarès & 102 & 15 & 7 & 7 \\
\hline
\end{tabular}


Table 2. Value of $p_{1}, p_{2}, p_{\min }$ and $p_{\max }$ for the 5 classes.

\begin{tabular}{lllll}
\hline $\begin{array}{l}\text { Pressure } \\
\text { class }\end{array}$ & $p_{1}$ & $p_{2}$ & $p_{\min }$ & $p_{\max }$ \\
\hline Very good & -6.00 & 0.65 & -6.00 & 25.00 \\
Good & 0.65 & 6.72 & -6.00 & 25.00 \\
Medium & 6.72 & 12.81 & -6.00 & 25.00 \\
Bad & 12.81 & 18.90 & -6.00 & 25.00 \\
Very Bad & 18.90 & 25.00 & -6.00 & 25.00 \\
\hline
\end{tabular}

Table 3. List of selected candidate metrics and corresponding characteristics of the pressureimpact GLMs.

\begin{tabular}{|c|c|c|c|}
\hline Type of metrics & Metrics & Definition & $\begin{array}{l}\text { GLMs: Family, link } \\
\text { function }\end{array}$ \\
\hline \multirow[t]{2}{*}{ Fish assemblage } & $\mathrm{SR}$ & Total species richness & Poisson, log \\
\hline & TD & Log (Total number of captured fishes +1 ) & Gaussian, identity \\
\hline \multirow[t]{10}{*}{ Ecological guilds } & NMIG & Number of distinct migrant species & Poisson, log \\
\hline & DMIG & Log(number of migrant fishes +1 ) & Gaussian, identity \\
\hline & NFW & Number of distinct freshwater species & Poisson, log \\
\hline & DFW & Log(number of freshwater fishes +1 ) & Gaussian, identity \\
\hline & NMJJ & Number of distinct marine juveniles species & Poisson, log \\
\hline & DMJ & Log(number of marine juveniles fishes +1 ) & \\
\hline & NMS & Number of distinct marine seasonal migrants species & Poisson, log \\
\hline & DMS & $\log ($ number of marine seasonal migrants fishes +1 ) & Gaussian, identity \\
\hline & NM & Number of distinct marine species & Poisson, log \\
\hline & $\mathrm{DM}$ & $\log ($ number of marine fishes +1 ) & Gaussian, identity \\
\hline \multirow[t]{4}{*}{ Trophic } & NNIB & $\begin{array}{l}\text { Number of distinct benthic invertebrate predators } \\
\text { species }\end{array}$ & Poisson, log \\
\hline & $\mathrm{DIB}$ & $\begin{array}{l}\text { Log (number of benthic invertebrate predators fishes + } \\
\text { 1) }\end{array}$ & Gaussian, identity \\
\hline & NF & Number of distinct fish feeders species & Poisson, log \\
\hline & DF & $\log ($ number offish feeders fishes +1 ) & Gaussian, identity \\
\hline \multirow{2}{*}{$\begin{array}{l}\text { Vertical } \\
\text { distribution }\end{array}$} & NB & Number of distinct benthic species & Poisson, log \\
\hline & $\mathrm{DB}$ & $\log ($ number of benthic fishes +1 ) & Gaussian, identity \\
\hline
\end{tabular}


Table 4. Kept candidate metrics and corresponding pressure-impact models, pressure index regression parameter and corresponding $p$-value

\begin{tabular}{|c|c|c|c|c|}
\hline Metric & Definition & Model & Regression parameter & $\rho$-value \\
\hline $\mathrm{SR}$ & Total species richness & Sal Class + Season $+\mathrm{Pr}-$ & -0.04 & 0.01 \\
\hline TD & Log (Total number of captured fishes +1 ) & Sect+Pr & -0.32 & 0.00 \\
\hline NM & Number of distinct marine species & Sect + Surf $+P r$ & -0.16 & 0 \\
\hline DM & $\log ($ number of marine fishes +1 ) & $\operatorname{Pr}$ & -0.12 & 0.05 \\
\hline
\end{tabular}

\section{Figures}

Figure 1. Maps of the different lagoons considered in this study.

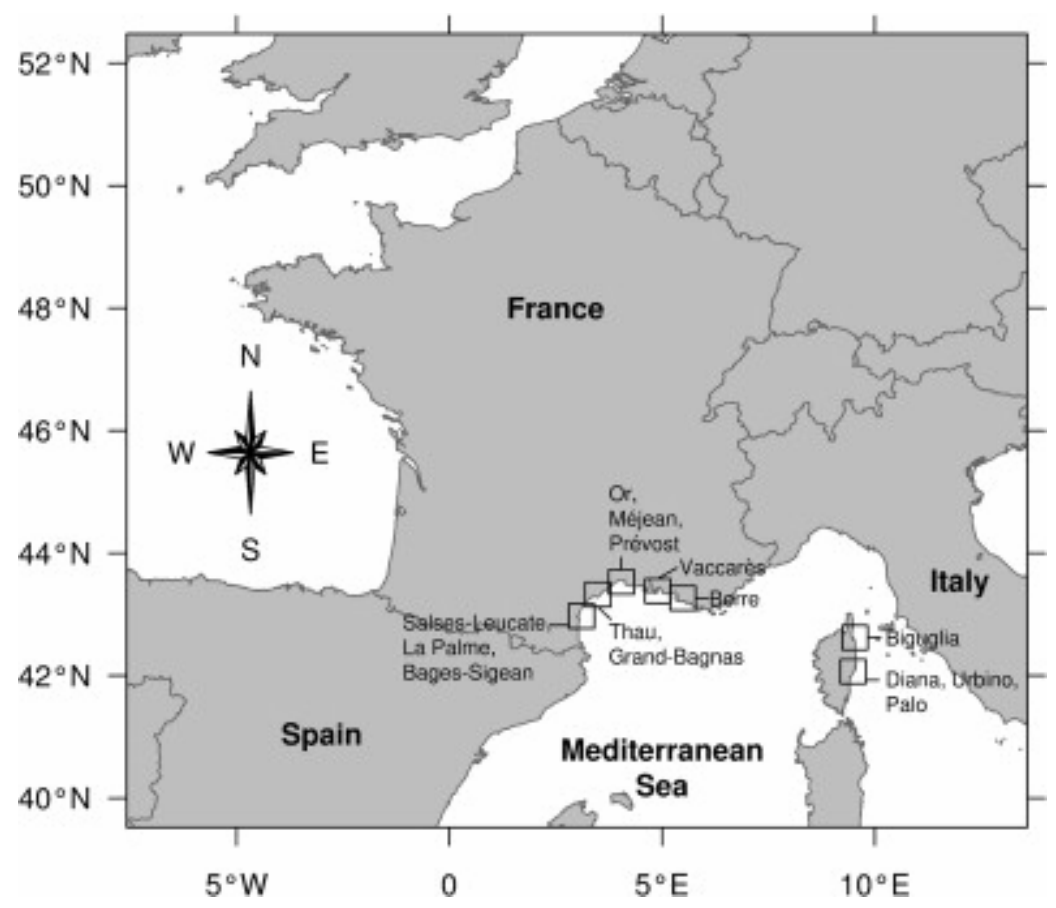

Figure 2. Calculated anthropogenic pressure index for each lagoon (a high PCA coordinate implies a high level of pressure) and corresponding quality classes ("very good, "good", "medium", "poor","very poor") thresholds (dotted lines).

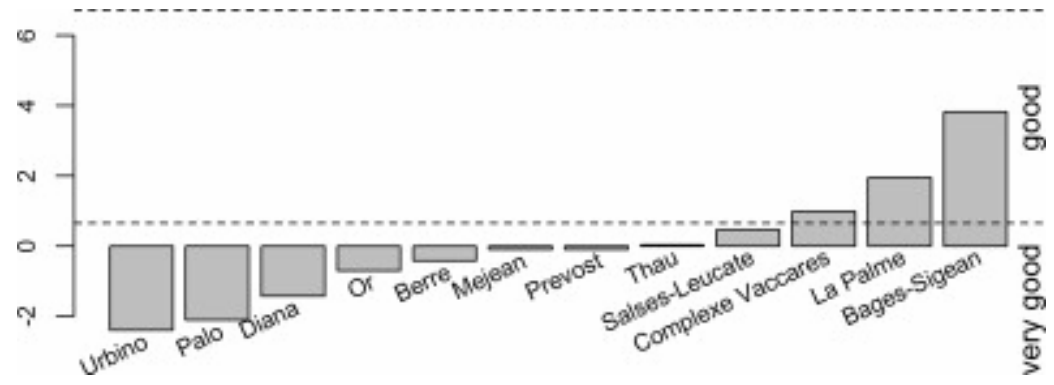


Figure 3. Posterior probability to be in a quality class given the observations (barplot) and pressure index quality class (from RINBIO, vertical bold line).

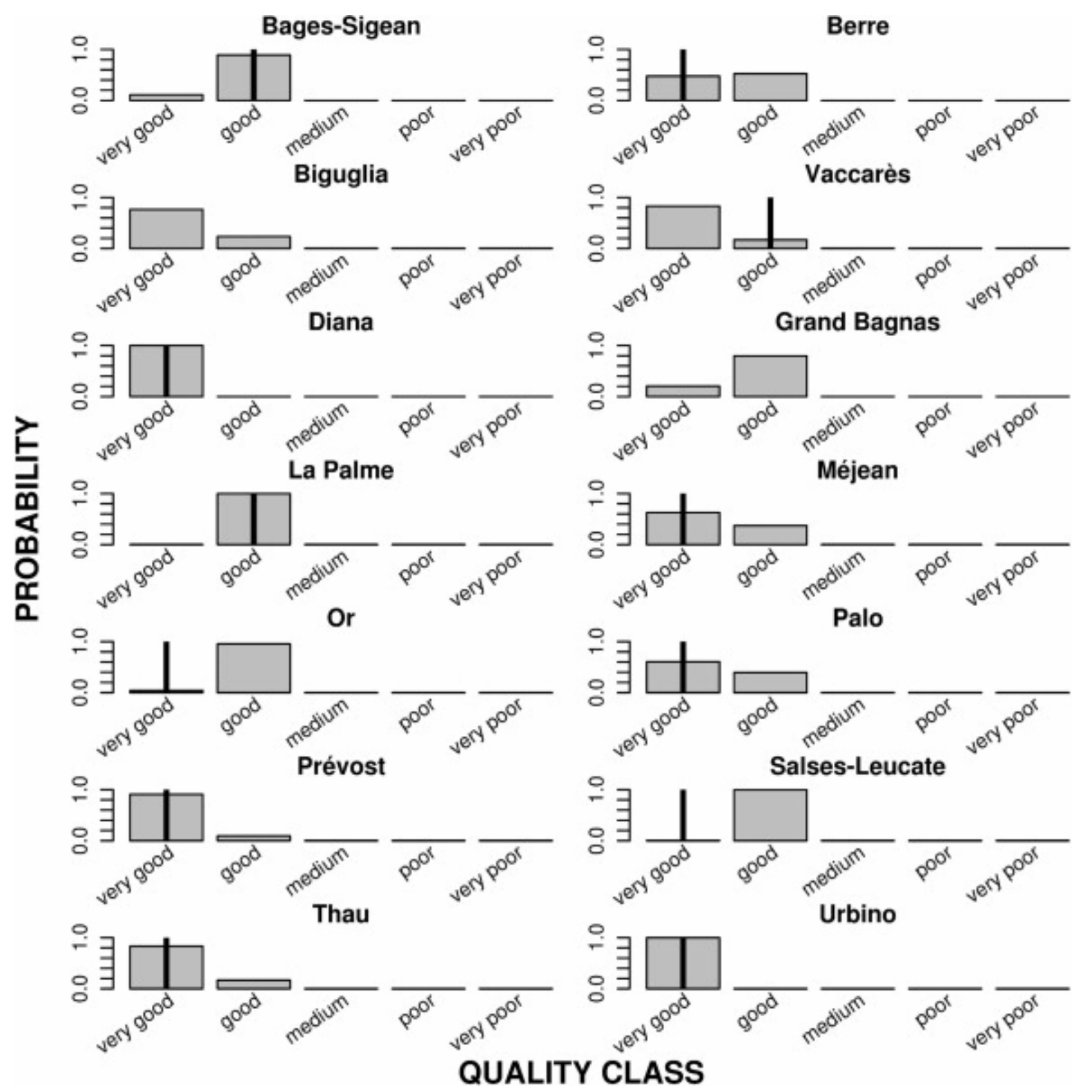


Figure 4. Posterior probability to be in a quality class given TD (white bars), DM (light grey bars), RT (dark grey bars) considered individually, and pressure index quality class (from RINBIO, vertical bold line).

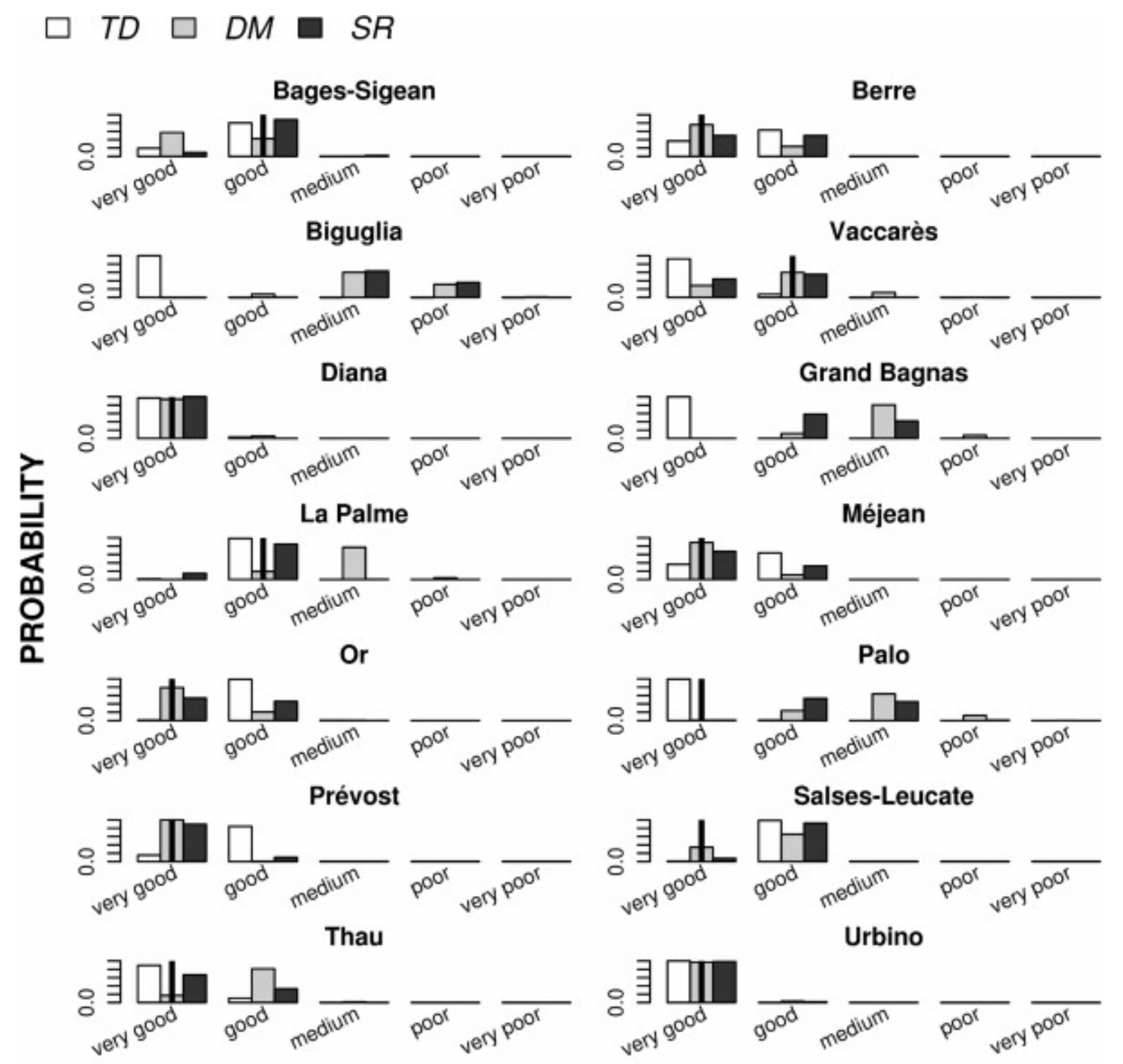

